\title{
Philosophiques
}

\section{Réalisme contextuel et perception sensible. Commentaires sur Sens et sensibilité de J. Benoist}

\section{Denis Fisette}

Volume 37, numéro 2, automne 2010

URI : https://id.erudit.org/iderudit/045195ar

DOI : https://doi.org/10.7202/045195ar

Aller au sommaire du numéro

Éditeur(s)

Société de philosophie du Québec

ISSN

0316-2923 (imprimé)

1492-1391 (numérique)

Découvrir la revue

Citer ce document

Fisette, D. (2010). Réalisme contextuel et perception sensible. Commentaires sur Sens et sensibilité de J. Benoist. Philosophiques, 37(2), 483-490.

https://doi.org/10.7202/045195ar d'utilisation que vous pouvez consulter en ligne.

https://apropos.erudit.org/fr/usagers/politique-dutilisation/ 


\title{
Réalisme contextuel et perception sensible. Commentaires sur Sens et sensibilité de J. Benoist*.
}

\author{
DENIS FISETTE \\ Université du Québec à Montréal
}

Sens et sensibilité. L'intentionalité en contexte se veut une série d'exercices sur "l'intentionalité en contexte », dont le fil conducteur est la cause réaliste que Jocelyn Benoist cherche à défendre en dialogue avec plusieurs philosophes (de Husserl à C. I. Lewis en passant par W. James, M. Schlick, J. Austin, H. Putnam, etc.) et dans le contexte de problématiques très diversifiées (de la perception sensible au débat opposant le réalisme à l'anti-réalisme). La thèse générale formulée dans la préface de l'ouvrage est que le « réalisme contextuel » est « l'envers du réalisme intentionnel» (p. 6). Benoist n'a pas cru bon d'expliciter les présupposés généraux de cette thèse, ni de rédiger une introduction générale qui aurait pu guider le lecteur dans sa lecture de cet ouvrage complexe et labyrinthique. Le lecteur est donc remis à lui-même, et, pour s'y retrouver, il n'a d'autre choix que de s'instruire des travaux antérieurs de Benoist et de rechercher les repères lui permettant de progresser dans sa lecture de l'ouvrage. J'associerai d'entrée de jeu l'intentionalité à la théorie de Husserl, comme le suggèrent d'ailleurs fortement les travaux antérieurs de Benoist, et le réalisme contextuel au réalisme (interne ou naturel) de H. Putnam, comme semblent le confirmer les derniers chapitres de l'ouvrage. La question est de savoir comment se conjuguent intentionalité et réalisme, plus précisément le domaine des phénomènes mentaux dont le trait caractéristique est d'être à propos des objets, et celui de la réalité du monde environnant auquel appartiennent les objets de ces actes. J'examinerai le thème de l'intentionalité en relation avec les analyses de la perception et de l'action dans les deux premières parties de l'ouvrage, et je terminerai par quelques remarques critiques sur la forme de réalisme esquissée dans la dernière partie.

\section{L'intentionalité de la perception}

Le premier chapitre porte sur la théorie de la perception du jeune Husserl, et Benoist s'appuie sur quelques-uns des écrits du philosophe de Halle rédigés entre 1893 et 1899 , et publiés à titre posthume. Rétrospectivement, je dirais qu'il s'agit du chapitre le plus original et le plus important. On remarque, comme dans ses derniers ouvrages, une certaine ambivalence de la part de Benoist face à la phénoménologie husserlienne. D’un côté, il semble vouloir défendre Husserl contre certaines critiques insistantes depuis

* J. Benoist Sens et sensibilité. L'intentionalité en contexte, Paris, Cerf, 2009. 
Merleau-Ponty, qui lui reprochent son intellectualisme (perception $=$ jugement). Elles font valoir avec raison que plusieurs des idées qu'on oppose à un certain Husserl, principalement celui des ouvrages publiés de son vivant, se retrouvent dans les écrits d'un autre Husserl, qui est demeuré dans l'ombre du premier et qu'ont exploité depuis quelques années de nombreux philosophes se réclamant aussi de Merleau-Ponty. Mais d'un autre côté, il lui reproche son "intentionnalisme ", c'est-à-dire une conception de la perception sensible se réduisant à une "forme d'intentionalité » (p. 17) qu'il critiquera dans les chapitres suivants. Contre l'intentionnalisme, Benoist soutient qu'une théorie viable de la perception doit reconnaître «la part du nonintentionnel dans l'intentionnel ", c'est-à-dire, comme il est précisé à la fin de la première partie (p. 101), que l'on doit rendre compte du fait que la perception est sensible "au » monde ou «à » certains aspects du monde, ce qui implique en retour que l'agent soit "réellement» en relation avec les choses de son monde environnant. La question sous-jacente à ce premier chapitre est de savoir si et comment une théorie de la perception, notamment celle du jeune Husserl, peut satisfaire à ces exigences.

Les textes de Husserl analysés dans ce premier chapitre suggèrent non seulement qu'il avait bien vu ce problème, mais qu'il propose une solution semblant répondre aux exigences que Benoist impose à une théorie de la perception sensible. Partons de la fameuse distinction entre «contenu réel » et «contenu intentionnel» d'un acte de perception. Cette distinction s'est imposée à Husserl dans le contexte d'une critique de la théorie de l'intentionalité de Brentano et de son étudiant $\mathrm{K}$. Twardowski, et Benoist a bien vu (p. 30) que la théorie de l'intentionalité élaborée par Husserl au milieu des années 1890 est aussi redevable des travaux de son maître C. Stumpf à Halle sur la notion d'attention, laquelle est conçue dans sa Psychologie du son comme intérêt ou «Lust am Bemerken». La notion de «contenu réel » de la perception tire son origine de la critique que Husserl adresse à la doctrine brentanienne des phénomènes physiques dans ses premiers travaux à Halle, et elle correspond à ce qu'il appelle les contenus primaires. En effet, dans le chapitre III de Philosophie de l'arithmétique, Husserl propose de remplacer la notion brentanienne de phénomène physique par la notion centrale de «contenu primaire» sur laquelle repose sa propre conception des sensations dans les Recherches logiques ${ }^{1}$. Husserl soutient que ces contenus primaires sont des touts structurés par des relations (les relations fondatrices de Stumpf), notamment par les relations de fusion et les relations méréologiques, lesquelles sont au cœur de la théorie des touts et des parties qu'il élabore dans sa troisième Recherche.

Cette distinction cardinale entre contenu primaire et acte psychique, quoique marginale dans Philosophie de l'arithmétique, représente un acquis dans les ouvrages ultérieurs de Husserl, et elle joue un rôle central dans la

1. Cf. Husserl, Philosophie de l'arithmétique, Paris, PUF, 1972, p. 86. 
phénoménologie des Recherches logiques. On la retrouve notamment dans ses deux études publiées en 1894 sous le titre «Études psychologiques pour la logique élémentaire» et, comme l'a bien vu Benoist, elle est le thème central d'un traité publié récemment sous le titre "Abhandlung über Wahrnehmung von 1898 ", lequel était vraisemblablement destiné à la deuxième série des Recherches logiques ${ }^{2}$. Son point de départ est justement la double confusion dans l'usage de la notion de phénomène (= contenu de sensation) par les psychologues et scientifiques de l'époque entre, d'une part, le contenu et l'objet de la perception, d'autre part, entre percevoir et sentir. Dans la sixième Recherche (p. 87), Husserl reconnaît à ces contenus primaires le statut de genre supérieur et leur assigne un rôle fondateur dans la hiérarchie des contenus psychiques. Ces contenus primaires désignent en effet les contenus de la sensibilité «externe » et forment "un genre suprême unique ". Par cette distinction entre contenu primaire et acte psychique, Husserl semble vouloir échapper à l'objection représentationnaliste ou intentionnaliste qu'il adresse explicitement à Brentano durant la période de Halle, lorsqu'il fait valoir que le principe suivant lequel tout phénomène psychique est ou bien une représentation, ou bien a une représentation pour base ne s'applique qu'à la classe des vécus intentionnels. À l'autre classe de l'expérience sensible qui n'obéit pas à ce principe appartiennent le plaisir et le déplaisir sensibles de même que les fameuses sensations affectives dont nous reparlerons plus loin. Ces modes de conscience non intentionnels, que Husserl appelle parfois "conscience primaire» ou encore intuition, interprétation, appréhension et aperception, et qu'il associe étroitement aux contenus primaires, sont les plus primitifs et occupent le niveau inférieur ou soubassement dans cette hiérarchie.

Non seulement Husserl reconnait-il la part du non-intentionnel (contenu primaire ou réel) dans sa première théorie de la perception, mais la tâche principale qu'il assigne à sa phénoménologie dans la première édition des Recherches logiques se confond avec l'analyse et la description de ces contenus réels, comme le confirment de nombreux passages, dont celui-ci:

En fait, le mot "phénoménologique » comme aussi le mot «descriptif» avaient été, dans la première édition de ce livre, entendus exclusivement comme se rapportant à des composantes réelles de vécus, et même, dans la présente édition, nous les avions jusqu'ici employés de préférence dans ce sens. Ce qui est conforme au fait que le point de départ naturel de nos recherches se trouvait dans l'attitude psychologique (cinquième Recherche, p. 202, note 1).

Comme nous l'avons vu précédemment, «réel» ne s'applique ici qu'aux contenus primaires, et il s'oppose à la fois aux vécus intentionnels et aux composantes physiologiques ou génétiques des sensations correspondantes. Or une des tâches principales de la phénoménologie des Recherches logiques

2. Husserl, Hua XXXVIII, pp. 123-158. 
réside précisément dans l'analyse de ces contenus réels et primaires. Si tel est bien le cas, alors la phénoménologie échappe aux objections d'intentionnalisme et à ce que $j$ 'appellerais la thèse du caractère coextensif de la conscience, de l'intentionalité et de la pensée. Car ces contenus primaires sont associés à un mode de conscience qui n'est pas intentionnel et correspond à ce qu'on appelle depuis quelque temps la conscience ou l'expérience phénoménale. Ce mode de conscience, qui a plus d'extension que la conscience intentionnelle qui le présuppose, entretient avec le monde environnant une relation particulière dont l'analyse ne ressortit pas à une théorie de l'intentionalité.

\section{L'intentionalité mise en cause}

Le deuxième chapitre est consacré aux travaux de J. Austin sur la perception. Il est difficile d'entrée de jeu de voir en quoi consiste la contribution de ce philosophe des actes de langage dans l'économie de la première partie de l'ouvrage. Benoist reconnaît qu'il n'y a pas d'intentionalité dans la théorie de la perception d'Austin, et je dirais que ses analyses se situent à un autre niveau que celles de Husserl ou encore de celles qui sont en cause dans certains débats contemporains sur la perception (je pense ici au débat opposant conceptualisme et non-conceptualisme). En effet, ce que Austin appelle Ways of seing de même que ses descriptions sont en fait des modes de description conceptuels - ou sémantiques puisque ces descriptions sont linguistiques - qui ne contribuent en rien à l'analyse de la perception sensible en tant que telle, mais la présupposent. Benoist semble impressionné par l'idée que ce genre de descriptions varie en fonction du contexte et des circonstances des agents linguistiques, mais ne s'agit-il pas alors d'une redescription appartenant à l'espace des concepts, pour reprendre une expression à la mode? Il en va autrement des modes de donation d'un seul et même objet perçu qui, comme le montre le premier chapitre de l'ouvrage, appartiennent au contenu réel (non intentionnel et non conceptuel) de la perception et rendent possible une relation directe avec le monde environnant. $\mathrm{Ne}$ doit-on pas admettre, avec le réalisme naturel, que ce sont ces modes de donation et non les modes de description conceptuels qui nous rivent, comme le veut Benoist, «à la présence du monde dans son épaisseur» (p. 51)?

Dans le chapitre III, Benoist reprend la question initiale dans le domaine de la morale et soutient, en s'appuyant sur une interprétation des leçons de Husserl sur l'éthique, que les jugements moraux doivent être fondés sur la perception "ordinaire» (p. 96-97). Tout dépend bien entendu de ce qu'on met dans cette classe d'acte et dans la relation de fondation que l'on établit entre le contenu (intentionnel) de ces actes et la perception sensible ou, pour reprendre le vocabulaire des Recherches logiques, les contenus primaires ou réels. Ces derniers correspondent à ce que Husserl appelle dans la $\$ 15$ de la cinquième Recherche les Gefühlsempfindungen, ou sensations affectives; ils appartiennent à la classe des contenus primaires et entretiennent avec les actes de la classe des sentiments une relation similaire à 
celle de la perception externe avec les contenus sensibles. Telle est la thèse que Husserl oppose à la doctrine des émotions de Brentano dans ses Recherches logiques. Dans cette étude minutieuse de la doctrine brentanienne des sentiments, Husserl dénonce d'entrée de jeu une confusion sur le plan conceptuel dans l'usage de la notion de sentiment (Gefübl) entre sensation affective (Gefühlsempfindung) et acte de sentiment (Gefühlsact), entre les sensations affectives appartenant au domaine des phénomènes physiques, et les émotions ou actes de sentiment que Brentano, par exemple, range dans la troisième classe des phénomènes psychiques. L'objet du litige se présente d'abord comme la question de savoir si les douleurs et les plaisirs corporels, les sentiments liés aux sensations des sens spécifiques comme la température, le bruit, le goût, le son, la couleur ou même le plaisir que procure une œuvre d'art sont de nature intentionnelle, au même titre que la joie, la tristesse, la colère, l'espoir, l'envie, le dégoût, comme le veut Brentano, ou bien de nature sensorielle ou phénoménale comme le soutiennent les sensualistes, tel W. James. La question de savoir si des sentiments comme le plaisir et le déplaisir, la douleur ou la jouissance esthétique appartiennent à la classe des phénomènes physiques ou bien à la classe des phénomènes psychiques au même titre que la joie et la tristesse, ou encore que tous les phénomènes liés au désir et à la volonté. La position que défend Husserl dans la $\$ 15$ de la cinquième Recherche repose encore ici sur la distinction entre contenus primaires et actes psychiques, et elle consiste à ranger plaisirs et douleurs, par exemple, dans la classe des contenus sensibles, tout en reconnaissant avec Brentano et contre les sensualistes que les émotions comme la honte ou l'envie appartiennent à la classe des actes intentionnels. Gefüblsackte et Gefüblempfindungen appartiennent à deux genres descriptifs différents, à savoir à celui des phénomènes psychiques pour les actes du sentiment, et à celui des contenus primaires dans le cas des Gefühlsempfindungen. En tant que contenus sensibles, les sensations affectives ont une fonction équivalente pour les actes de sentiment à celle des contenus présentatifs pour la perception externe, et elles présupposent donc une certaine interprétation ou appréhension que je ne commenterai pas ici. La joie ou la tristesse, le désir ou la volonté présupposent à la base une aperception du contenu sensible sur lequel ils se fondent.

Même en admettant le rôle fondateur de la perception sensible, et plus précisément des sensations affectives dans le domaine de l'éthique, la question de la structure des jugements moraux, c'est-à-dire de la classe des actes spécifiques au domaine de la morale, demeure entière. À propos des valeurs par exemple, doit-on supposer que ce sont des contenus intentionnels de la classe (des actes) des sentiments, qu'ils ont un statut équivalent à celui des propositions ou des contenus de jugement et qu'ils ont pour objets des Wertverhalten, c'est-à-dire l'équivalent des états de choses dans le domaine du jugement? Le chapitre XI sur le réalisme moral, qui porte à nouveau sur Husserl, nous informe seulement sur ce que les valeurs ne sont pas, à savoir 
des particuliers abstraits comme le veulent certains phénoménologues réalistes (p. 286-287). Ce qui est clair en revanche, c'est que Benoist opte pour une forme de réalisme des valeurs morales qu'il cherche à défendre contre deux versions concurrentes de la phénoménologie réaliste: l'objectivisme et l'ontologisme. Je retiens deux arguments de Benoist contre ces dernières: le premier est contextualiste, et il repose sur l'idée que c'est le contexte ou la situation qui confèrent un sens aux valeurs (p. 288); le deuxième consiste à dénoncer un présupposé de ces deux versions du réalisme moral, à savoir celui d'une intentionalité d'objet qui serait responsable d'une certaine cécité face à "la diversité de l'activité intentionnelle de sujets dans le monde en tant qu'acteurs dans des situations déterminées» (p. 291). J'examinerai brièvement ces deux arguments en relation à ce qu'il appelle, dans la troisième partie de l'ouvrage, la pragmatique intentionnelle dans laquelle il aborde le thème de l'action et affirme la primauté de la raison pratique sur la raison théorique.

Le chapitre VI intitulé «Prothèses » porte sur l'intentionalité de l'action, plus précisément sur la philosophie de l'action dans laquelle la notion d'intention joue un rôle central. La notion de prothèse désigne ici les facteurs extérieurs et non intentionnels à l'action, c'est-à-dire encore une fois la situation de l'agent et le contexte sociologique, culturel, institutionnel et causal qui déterminent le sens de l'action. Benoist utilise le cas des chaînes causales déviantes afin de critiquer la définition de l'action comme comportement intentionnel et de démontrer que le comportement se fonde sur du non-intentionnel. Là encore, Benoist n'a pas cru utile de fournir une analyse détaillée de la structure de l'action qu'il critique dans ce chapitre, et notamment de la théorie de l'intentionalité sur laquelle elle s'appuie. Admettons qu'elle soit connue, la question est alors de savoir si le cas des chaînes causales déviantes représente un problème sérieux pour une théorie standard de l'action. Partons du cas suivant que Benoist reprend de Davidson: "A tire sur B pour le tuer. Mais c'est un mauvais tireur. Il rate sa cible, mais la détonation met en branle un troupeau de phacochères, dont la charge écrase la cible, qui meurt» (p. 167-168).

Le troupeau de phacochères agit dans ce cas comme «prothèse » en tant que moyen indirect ou véhicule de l'action, et Benoist soutient qu'il ne s'agit pas là d'un cas d'exception mais bien de la règle qui gouverne le comportement en général. Cette conclusion ne me semble pas entièrement justifiée, tout d'abord parce que les cas de chaînes causales déviantes ne concernent en définitive que l'efficience causale de l'intention sur l'action, ou encore de l'action sur les résultats escomptés. Mais ils ne mettent aucunement en question le caractère intentionnel du comportement, ils montrent au contraire que l'intentionalité de l'action n'est pas fonction de ses résultats (l'action de A de tirer un coup de feu). Il faut également distinguer l'action comprise comme un comportement intentionnel de l'intention comprise comme un état mental au même titre que le désir ou la croyance dont le trait caractéristique essentiel est 
l'intentionalité (l'intention vise le résultat escompté). Dans un cas comme dans l'autre, c'est-à-dire dans son sens pratique comme dans son sens technique, l'intentionalité ne dépend pas des conséquences de l'action. Que certaines situations dans le monde environnant déterminent de manière "causale » la plupart de nos comportements, c'est là une question différente, comme le montre une version légèrement modifiée du cas utilisé par Benoist. Supposons en effet que B menace A en pointant son arme vers lui. En légitime défense, $\mathrm{A}$ forme l'intention d'atteindre $\mathrm{B}$ à l'aide de son arme et tire en sa direction, etc. La notion de motivation que Benoist utilise au début de ce chapitre (p. 162) est tout à fait appropriée dans le cas précis où il s'agit de rendre compte du «parce que» de l'action en faisant intervenir des facteurs non intentionnels qui la déterminent de manière quasi causale (A est motivé par la représentation qu'il se fait de B dans le contexte X). Cela dit, le recours à ces facteurs externes ne met pas en cause la nature intentionnelle de l'action, mais seulement une conception particulière et erronée de l'intentionalité (voir le chapitre IX sur le débat internalisme-externalisme).

\section{Sur le réalisme intentionnel}

Pour terminer, je commenterai brièvement la cinquième et dernière partie de l'ouvrage consacrée au réalisme intentionnel, et plus spécifiquement le chapitre $\mathrm{X}$ qui porte sur les différentes versions du réalisme défendues par Putnam au cours des dernières années. Le sens même de la notion de "réalisme intentionnel » ne va pas de soi et soulève une question méthodologique centrale dans la tradition phénoménologique. Car, d'une part, une théorie intentionnelle est distincte des questions métaphysiques sur l'ameublement du monde extérieur, et, d'autre part, étant donné ses postulats méthodologiques de base, la phénoménologie n'admet pas que l'on puisse statuer $a$ priori sur ces questions métaphysiques en associant d'entrée de jeu intentionalité et réalisme. Benoist reconnaîtra sûrement ici deux principes qui sont à l'œuvre dans la phénoménologie des Recherches logiques de Husserl: le premier est le principe d'absence de présupposition métaphysique, tandis que le deuxième stipule que la phénoménologie doit prendre appui sur «les modes de donation véritables des phénomènes ". Le premier principe impose à la phénoménologie le principe méthodologique de la neutralité, c'est-à-dire l'absence de présupposition concernant l'existence et la nature du monde extérieur de même que les lois physiques qui la sous-tendent. Husserl soutient en effet que l'on ne peut statuer a priori sur ces questions métaphysiques parce que celles-ci présupposent justement certaines analyses de la phénoménologie. Le deuxième principe, que l'on pourrait qualifier d'empiriste au sens de James par exemple (voir chapitre IV), stipule que le critère non métaphysique servant à distinguer entre le domaine de l'expérience et le monde des objets transcendants doit prendre appui sur le "caractère descriptif des phénomènes tels qu'ils sont vécus ", c'est-à-dire justement sur 
cette couche originaire de l'expérience des contenus primaires qui, dans la phénoménologie, fait fonction de tribunal de l'expérience.

C'est ce qu'a bien vu H. Putnam dans The Threefold Cord: Mind, Body, and World (New York, Columbia University Press, 1999) quand il interprète les thèses de Husserl sur la Lebenswelt en disant que les qualités primaires de la tradition ne sont en définitive que des "abstractions idéalisées » et que "there is nothing "secondary" about such experienced attributes of things as color» (p. 38-39). Le réalisme naturel que Putnam attribue aussi bien à W. James qu'à Husserl veut que ces attributs, qui appartiennent aux contenus réels et primaires de la perception, soient directement en relation avec les aspects des choses correspondantes dans le monde extérieur (p. 24). Quelle est la nature de ces relations? Le cas des chaînes causales déviantes semble suggérer que Benoist les conçoit comme des relations causales singulières. Si tel est bien le cas, comment alors ce postulat cadre-t-il avec ce qu'il appelle le réalisme contextuel et le réalisme intentionnel? À défaut d'une réponse claire à ces questions, l'ouvrage ne me semble pas offrir une véritable option à ce qu'il appelle les «métaphysiques analytiques» (p. 272275) qui, pour être critiquables, représentent néanmoins une option qui a fait ses preuves dans l'histoire de la philosophie depuis Brentano et au-delà. 